\title{
Modular Platforms for Optofluidic Systems
}

\begin{abstract}
Optofluidics is increasingly gaining impact in a number of different fields of research, namely biology and medicine, environmental monitoring and green energy. However, the market for optofluidic products is still in the early development phase. In this manuscript, we discuss modular platforms as a potential concept to facilitate the transfer of optofluidic sensing systems to an industrial implementation. We present microfluidic and optical networks as a basis for the interconnection of optofluidic sensor modules. Finally, we show the potential for entire optofluidic networks.
\end{abstract}

\section{Keywords}

optofluidics $\cdot$ microfluidics $\cdot$ micro total analysis systems • backplane $\cdot$ modular platform

PACS:

(C) Versita sp. z o.o.

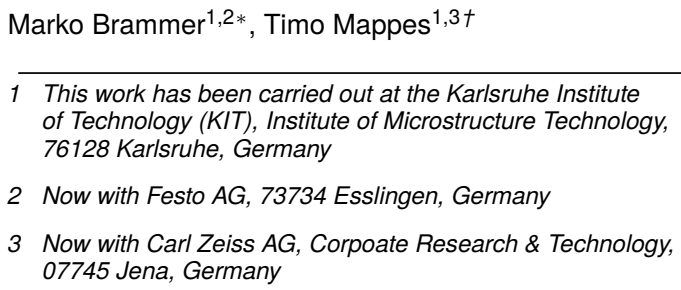

Received 2012-12-21

Accepted 2013-01-21

\section{Introduction}

Optofluidic systems are based on the interaction between light and fluid. Controlling the properties of the fluid allows for manipulating the properties of this interaction. Due to this significant advantage, considerable research progress has been made in the recent past towards complex system design for several applications [1-6]. One major research focus lies on analysis applications in areas such as pharmaceutical, biotechnology, and life-sciences.

This progress in optofluidics is comparable with the development of microfluidics in the 80 s and 90s [7-9]. First microfluidic devices have been fabricated by well-proven microelectromechanical systems (MEMS) technologies in silicon and glass [10-12]. In contrast to most MEMS devices, microfluidic devices are larger and often used as disposables. Thus, new cost-efficient fabrication technologies and materials were crucial for commercial success. Henceforth, most devices were made of polymers and structured by replication technologies. The polydimethylsiloxane (PDMS) based soft-lithography process offers a method for rapid prototyping with low investment costs needed for implementtation [13, 14]. Moreover, PDMSbased devices inherit several advantages, such as tunability due to its viscoelastic behavior. This caused the widespread use of this technology platform for microflu-

*E-mail: marko.brammer@googlemail.com

†E-mail: timo.mappes@kit.edu idic demonstrators in the research society. Optofluidics emerged from microfluidics and thus uses mainly the same fabrication technologies.

However, soft-lithography has not been successfully established for large-scale industrial implementation yet, due to several drawbacks, such as swelling with several solvents and of permeability of dyes $[15,16]$. Until now industrial fabrication of microfluidic and miniature fluidic devices is mostly based on thermoplastic replication technologies $[17,18]$, such as injection molding, hot embossing or injection embossing [15, 19-21]. Examples for widely used thermoplastic materials are Polymethylmethacrylate (PMMA) and Cyclic-Olefin Copolymers (COC). Potential low-cost alternatives are cellulose-based materials $[22,23]$. The technologies of choice are still to be defined. This applies for structuring as well as for assembly and packaging of the fluidic channel plates. Candidates for assembly technologies are thermally, chemically, and radiation induced bonding, adhesive bonding [24], and laser beam welding [25].

Microfluidics and optofluidics have further aspects in common besides the fabrication technologies. To date the commercially most important industrial implementation of a MEMS-based microfluidic device is the ink-jet printer head [26]. Similarly to microfluidics, the commercially most relevant industrial implementation of an optofluidic principle, the liquid crystal display $[27,28]$, remains isolated without strong impact for other applications. Thus, both technologies, which were driven by large consumer 
markets, are not fully considered to be part of the corresponding research fields.

Several stand-alone sensor systems have been commercialized, based on fluorescence (Agilent, Affymetrix, Gyros, Biosite), absorption (Nanostream), colorimetry (Eppendorf Array Technologies), surface plasmon resonance (General Electrics Healthcare, Sensata) or interferometry (Fairfield). However, in 2001, a market volume in the field of microfluidics of 3 billion USD has been predicted for 2004 [29], of which however only $10 \%$ have been achieved. In 2011 the market volume of 3 billion USD was expected to be reached in 2014 [30].

A number of reasons may be considered, in order to achieve a progress in commercial growth, such as (1) missing well-defined coupling interfaces in between components and with the outer world [31], (2) use of different fabrication technologies and materials in research and industry [15], and (3) missing break-through applications [32].

In scientific projects there is the tendency to develop optofluidic on-chip screening platforms covering the fluidic channel size from $100 \mathrm{~nm}$ for single DNA molecule sequence mapping [33] to compact whole-animal on-chip imaging [34]. These approaches are expected to result in a number of commercial applications within the present decade.

One of the predominant success factors for industrial implementation is cost-efficiency. The system functionality and its application will define the approach to follow. For operating analysis systems, the sensor components need to be combined with switching and manipulating elements. In the development of these systems, two major design principles are applicable. Either all elements and functions are integrated into one individual device, or a set of modules is implemented that may be individually interconnected as required (Fig. 1). If high unit numbers are produced, a fixed configuration may be more cost-efficient. However, the modular design concept may be the better choice when developing a product family of similar system configurations.

Modular system design has its drawbacks in terms of functionality, since it is not optimized for each individual application. Examples are, (1) longer channel length, leading to slower dynamic response, and higher analyte and energy consumption. Furthermore, (2) modular systems necessitate more components, such as mechanical, optical, fluidic, and potentially electrical coupling interfaces, resulting in lower coupling efficiencies.

However, a modular approach may reduce development time and costs when implementing new variants of a product family. (1) New variants may be created by choosing different combinations, such that custom-made functions may be configured. Thus, only the new functional compo- nent needs to be developed. (2) By exchanging or adding modules in an operational system it may be used for a variety of applications and with different working parameters. Moreover, (3) even if for each individual variant only a low number of pieces is available, they add up with the number of different variants. Thus, components with standardized design and dimensions may be fabricated by large-scale technologies.

Establishing interconnection platforms as a basis for a product family of optofluidic analysis systems requires: (1) Switching concepts for selectively guiding fluid and light between a custom-made combination and configuration of optofluidic modules, (2) compact and defined interfaces assuring stable mechanical, leak-proof fluidic and low-loss optical coupling between the modules, (3) components are cost-efficiently mass-producible.

An interconnection platform may be either designed (1) modularly in itself (Fig. 1(b)), or (2) with a predefined number of slots (Fig. 1(c)), such that each sensor module is mounted on top of a backplane module that is individually interconnected with other backplane modules. The first concept needs not only coupling interfaces to the sensor modules but also to the neighboring backplane modules. The advantage is, however, that the arrangement and complexity of the system may be user-defined and variably adapted to the target application.

In this manuscript, we discuss modular concepts and implementations of microfluidic (section 2), optical (section 3), and optofluidic platforms (section 4). In particular, we compare our recently introduced platform, consisting of backplane modules with dimensions of $40 \times 40 \times 50 \mathrm{~mm}^{3}$ [35] with other technological solutions. We equipped the module interfaces with optical and fluidic connectors. Mechanical interconnection has been realized by reversibly detachable magnetostatic connectors. Each backplane module offers an interface with optical and fluidic connectors for a functional module, such as an optofluidic sensor. The backplane modules are built of three virtual layers: (1) a microfluidic backplane layer for guiding fluids selectively through fluidic channels and microvalves, and through the sensor modules, (2) an optical backplane layer for guiding light through optical fibers and switches to the sensor modules, and (3) an electronic backplane layer for controlling the active elements in the system, namely the microvalves and optical switches.

\section{Microfluidic Platforms}

Microfluidic networks in analysis systems are used to guide the fluid to be analyzed through channels to the interaction zones of the sensors. The fluid may be guided passively by capillary forces or diffusion. Higher flow rates and pressure differences may be achieved by ex- 
(a)

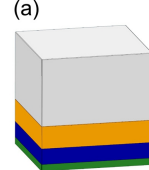

(b)

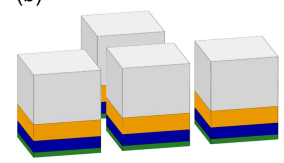

(c)

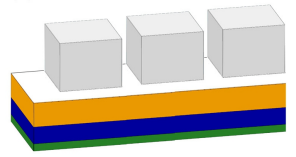

Fig 1. Generalized schematic of different system designs with microfluidic (blue), optical (yellow), and electronic (green) networking layers, and sensor units (white). (a) System with all components integrated in one device. (b) System with sensor modules interconnected by backplane modules. (c) System with sensor modules mounted on one backplane.

ternal pumping or by miniature pumps (e.g. Bartels Mikrotechnik mpx, bi.flow Systems, Bürkert 7604, Debiotech Nanopump) integrated into a compact system. Most miniature pumps are membrane-based, thus generating a pulsated fluid flow. Pulsation-free pumping for low flow rates is achievable by electroosmotic pumps (Osmotex, CSEM). Since the electrolysis produces gas, an intermediate element or fluid is necessary, limiting the range of possible applications. Available alternatives for higher flow rates are syringe pumps or pressure reservoirs with dosing valves, both being rather large and thus not compatible with compact portable devices.

In order to guide the fluid selectively through the channels without applying pressure differences, external or local fields may be used. By applying external electric or magnetic fields [36], or upon acceleration of the device, e.g., coriolis acceleration [37], the fluid may be guided along the chosen path. Alternatively, electrical fields locally applied by an electrode array are used, forming the basis of digital microfluidics devices [38]. Advantages of this principle include leveraging well-developed microelectronics fabrication technology, and the possibility to manipulate and analyze several individual fluid drops in parallel [39].

Higher pressures, however, require matching fluid flow control mechanisms. To fulfill this goal, integrated microvalves have been developed with several different designs and actuators [40]. They may be based on electrostatic, electromagnetic, piezoelectric or thermal actuation principles. Due to high energy density, shape memory alloy actuators are particularly interesting for compact design and have been developed in different variants [41, 42]. Drawbacks of these actuators are relatively high energy consumption and the limitation to operation temperatures below the transition temperature. For most materials the range is restricted to temperatures below $55^{\circ} \mathrm{C}$, which is not compatible with several process automation applications. Currently commercially available valves are mostly based on solenoids (Bürkert 6650, Kendrion Tri-Tech, LEE LFV, Staiger VA 204-7, Takasago VODA) and larger than
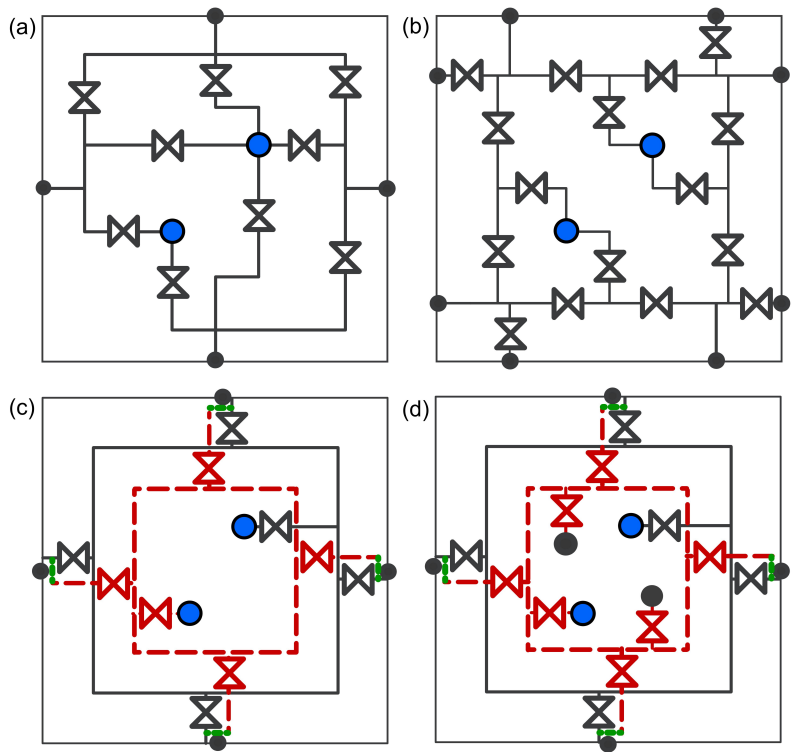

Fig 2. Schematics of microfluidic circuits consisting of fluidic channels and microvalves to be integrated into backplane modules. Fluidic connectors to the neighboring modules and a mounted device are marked with grey and blue circles, respectively. A second layer of fluidic channels and microvalves is illustrated in red, connecting channels in green. (a) Circuit with four connectors to neighboring modules in plane. (b) Circuit with eight connectors to four modules in plane. (c) Circuit with four connectors in plane and two layers (d) Circuit with connectors to six modules oriented in three dimensions and two independent layers.

$10 \times 10 \times 10 \mathrm{~mm}^{3}$, leaving potential for further development.

For high-density integration of microvalves, alternative concepts based on indirect actuation have been developed [43-45]. Two layers of microfluidic channels are built on top of each other and coupled either by an elastomeric intermediate membrane or two non-miscible fluids. The fluidic pressure in the first layer controls the switching states in the second layer, in which the analyte is guided. Whereas the microfluidic chip is very compact, a large external pneumatic control setup is needed. Thus, a modular system based on this principle would necessitate a large number of fluidic connectors.

Modular platforms have been presented [46-48] and are already available commercially from different companies (Microfluidic Chipshop, thinXXS Microtechnology), however without integrated fluid control devices.

We recently introduced the concept for fluidic circuits integrated into backplane modules and with coupling interfaces to devices to be mounted on top [49]. Depending on the circuit different flow paths are possible (Fig. 2). Microvalves mounted on top of the channel layer and controlled individually by an electronic circuit layer define the flow path between the coupling interfaces. Each mod- 


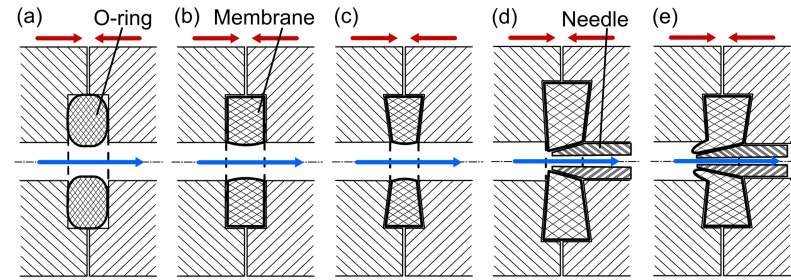

Fig 3. Schematics of fluidic coupling elements for module interconnection in cross-sectional views. Fluid flow is illustrated by blue arrows, compression force by red arrows. (a) O-ring. (b) Sealing membrane in a uniform notch. (c) Sealing membrane in a notch with inclined surface. (d) Needle inserted into a sealing ring [50]. (e) Needle pierced into a sealing membrane [51].

ule inherits interfaces to the neighboring modules and to the interconnected sensor module. The interface to the sensor module provides two channels to guide the fluid through the interaction zone of the sensor and back into the backplane layer. Depending on the circuit, the interfaces to the neighboring modules consist of one or two channels. Building two channel layers on top of each other enables intersection of two fluid flows without mixing. The fluidic network of choice for the dedicated application allows defined fluid control.

Another major issue to be solved is the development of coupling interfaces between small chips and the environment [31]. For modular systems connectors have to be integrated into the module interfaces, which is often done by O-rings or sealing membranes placed around the inlet of the fluidic channels (Fig. 3(a),(b)). A promising alternative concept for fluidic coupling is realized by hollow needles pressed into a tight fit elastomeric sealing ring [50], as illustrated in Fig. 3(d). Alternatively a non-structured sealing membrane is pierced by a needle (Fig. 3(e)), and sealed again due to internal stress of the elastomeric membrane when the needle is removed [51]. This design assures sealing in the disconnected state, it is, however, significantly more complex than O-rings or sealing membranes. Furthermore, low death volume and designs that may be easily flushed are important for clean and defined fluid flow. This requires coupling elements with well-defined channel boundaries as illustrated in Fig. 3(c). In this design the membrane is compressed at the borders of the fluidic channel by the inclined surface of the notch. Alternative solutions enable leak-proof coupling, but are often not designed symmetrically, which would limit the variability of interconnection of modules $[52,53]$.

Mechanical connectors compress the sealing elements and provide mechanical stability between the modules. However, the reliability of compact mechanical connectors is difficult to assure, particularly when they are designed for reversibly detachable interconnection $[35,54]$. We real- ized the mechanical interconnection between the modules by magnetic connectors. The magnetostatic force between a permanent-magnetic plug and a soft-magnetic socket is used to compress elastomeric sealing membranes at the coupling interfaces of the microfluidic backplane layers. Taking into consideration the influence of the magnetic field on the fluid flow, the fluid has to be insulated magnetically or cleaned from magnetic particles. We integrated the permanent-magnetic plug into a soft-magnetic holder, thus enclosing the magnetic field. This approach may have the potential to overcome the difficulties in coupling microfluidic circuits or modules with the system or lab environment.

\section{Optical Platforms}

Optical networks guide light from light sources to the interaction zones of the sensors, where the electromagnetic wave and the fluid overlap and the interaction properties are analyzed. Similarly to microfluidic platforms, one may differentiate between passive and active concepts. Passive concepts consist of optical waveguides or optical fibers, whereas active principles have additional controllable components, such as optical switches, multiplexers, and amplifiers.

Optical waveguides have been widely integrated into polymer chips and used as optical backplanes to guide light within sensor systems $[55,56]$. E.g., PMMA offers the possibility to integrate waveguides directly into the substrate by deep-ultraviolet radiation induced modification of the refractive index $[57,58]$. This process requires fewer fabrication steps than two-material waveguide fabrication.

Whereas for optical communication applications signal dispersion is crucial, optimization of waveguides for sensor systems is focused on efficiency. Low losses are possible with high contrast in refractive index [59]. In order to couple light into the waveguide, different approaches have been proposed. In particular, direct integration of light sources and waveguide into the same substrate allow for the most efficient coupling [60].

Optical fibers are an alternative to integrated waveguides. Since they are not integrated directly into the substrate, assembly costs are significantly higher. However, lower guiding and coupling losses are possible. Active switching between different fibers inside the optical network is mostly achieved by opto-mechanical switches. Several systems have been published $[61,62]$ and also integrated into commercial solutions (Lucent, DiCon, Fujitsu, Sercalo).

Recently, we presented optical backplane modules with integrated optical switches $[35,63]$. Five polymer optical fibers are inserted in each module, four of which are di- 

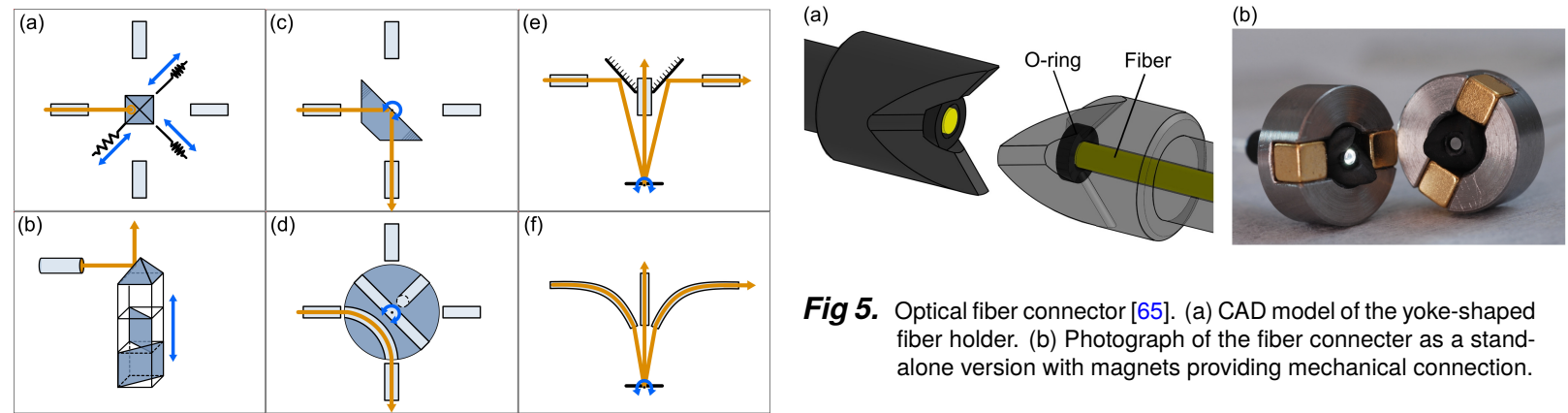

Fig 5. Optical fiber connector [65]. (a) CAD model of the yoke-shaped fiber holder. (b) Photograph of the fiber connecter as a standalone version with magnets providing mechanical connection.

Fig 4. Schematics of optical switches designed for five perpendicular orientated optical fibers. (a) Optical switch with three linearly actuated mirror elements (two double-sided plane mirrors, one pyramid mirror). (b) Linearly actuated optical switch as implemented in [63]. (c) Rotatory actuated optical switch with prism element, deflecting light by total internal reflection, mirror reflection or transmitting light. (d) Rotatory actuated optical switch with differently bent fibers. (e) MEMS mirror based optical switch with fixed pyramid mirror as implemented in [35] (f) MEMS mirror based optical switch with bent fibers.

rected horizontally to the neighboring modules and one vertically to the sensor module. The optical switch is positioned in the center of the module. Lenses are placed in front of each fiber. Light coupling out of one fiber is focused in the lens and coupled by the optical switch and through another lens into the chosen other fibers. Different opto-mechanical switching principles are illustrated in Fig. 4.

We developed two optical switches, each one optimized with respect to different parameters, (1) one with low coupling losses $(2.5-3.6 \mathrm{~dB})$ but long switching time (1 $3 \mathrm{~s})$, based on a linearly actuated assembly of optical elements [63] (Fig. 4(b)), and (2) another one with higher losses $(7.3-11.9 \mathrm{~dB})$ but faster switching ( $<30 \mathrm{~ms})$, based on an electrostatically actuated MEMS mirror [35] (Fig. 4(e)). Especially the performance of the MEMS mirror switch may be optimized, increasing the maximum angles of the quasi-static mirror deflection. This would lead to a shorter free-space distance between the fibers and thus to lower coupling losses. We used quasi-static deflection (Sercalo Microtechnology), since it offers the advantage of stable switching positions in comparison to resonant operation.

When coupling two modules, alignment is particularly important between the optical backplane layers, whereas the fluidic layers allow larger tolerances. In order to achieve mechanical alignment between fibers or waveguides, precisely shaped structures are used. Fabrication within such low tolerances is possible with lithographic-based techniques and subsequent replication technologies [64]. We assured precise alignment by yoke-shaped fiber holders that are mechanically self-aligned upon assembly (Fig. 5).

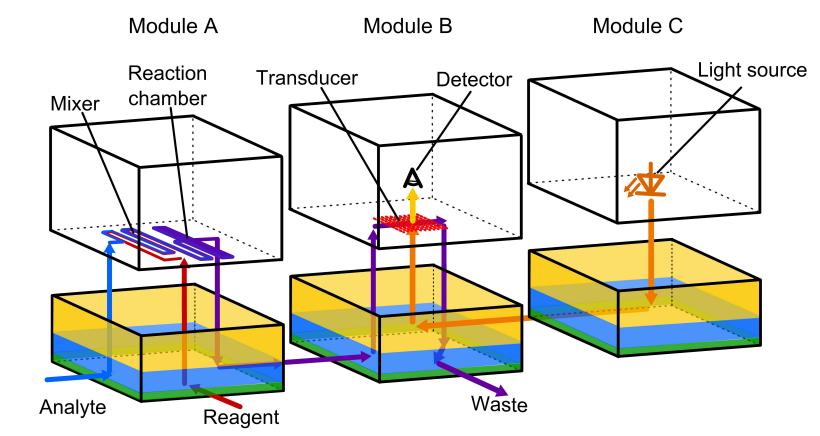

Fig 6. Scheme of an exemplary optofluidic analysis system based on the system design illustrated in Fig. 1(b) with microfluidic (blue), optical (yellow), and electronic (green) networking layers, and functional modules (white). The system consists of three backplane modules interconnecting one mixer/reactor $(A)$, one sensor (B), and one light source module (C). Light path is illustrated with orange arrows, analyte flow with blue, reagent flow with red, and mixed fluid with violet arrows.

The connectors are designed to be fabricated by micromachined tool fabrication and injection molding [65]. An O-ring provides the possibility to insert and seal an indexmatching gel.

\section{Optofluidic Platforms}

Based on modular microfluidic and optical platforms, optofluidic devices may be interconnected and operated. Fig. 6 shows a possible configuration of how to interconnect an optofluidic sensor module with backplane modules. We assembled microfluidic and optical backplane layers in one module, thus supplying different optical sensor modules with light and the fluid to be analyzed (Fig. 7) [35].

The proposed design is particularly advantageous for continuous monitoring of fluid flow parameters, crucial for several applications in life sciences, medicine, environmental, and process analysis [66, 67], as listed in the following. Optical and non-optical sensors operated in parallel would allow for measuring parameters, such as turbidity, color, the content of certain particles, the $\mathrm{pH}$ value or the oxygen content. The design holds the advantage that 

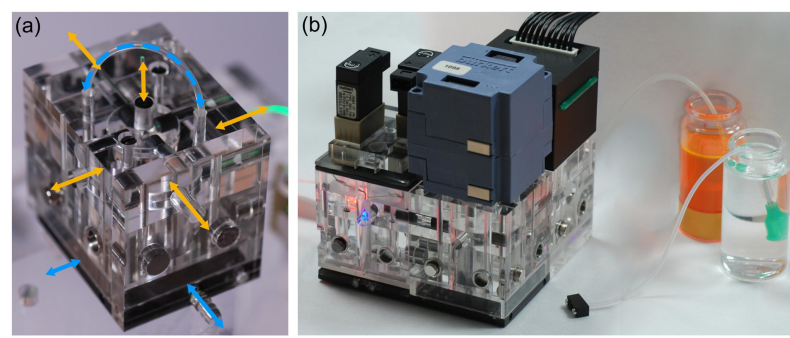

Fig 7. Photographs of the modular backplane [35]. (a) Prototype of the assembled optical and microfluidic backplane module $\left(40 \times 40 \times 50 \mathrm{~mm}^{3}\right)$. Fluidic connectors are marked with blue arrows, optical connectors with yellow arrows. The crossing of the tube and the fiber on top of the module illustrates the interface for an optofluidic device. (b) Measurement setup with three modules interconnecting a combined conductivity/temperature sensor module (Bürkert) and a miniature spectrometer (Hamamatsu C10988MA). The sensor modules are operated with a supply module with an integrated laser diode, two light emitting diodes, and two micropumps.

microfluidic and optical backplane layers are compatible but independently implementable. The functionality of the system may be chosen depending on the application, thus potentially reducing fabrication costs.

For some applications, a direct combination of optics and fluidics would be advantageous, when not only the sensor modules but also the platform itself is based on optofluidics. Different components have been presented that have the potential to be integrated into optofluidic analysis systems, with tunability being one of the most promising advantages. This would enable the control of the system parameters during operation, e.g., the measuring range of a sensor system may be adjusted to the environmental conditions.

One of the first optofluidic components were tunable lenses, of which the focal distance is controlled by changing the geometry or the refractive index $[68,69]$. The geometry of a liquid surface may be tuned by electrowetting [70-72] or an applied fluid pressure difference $[73,74]$. Lenses made from elastomeric polymers can also be modified by applying a pressure difference [75-77]. The refractive index can be controlled simply by changing or mixing the fluid. Alternative methods for manipulating geometry or refractive index are electrical, thermal or optical effects $[68,69]$. The high number of methods reflects the possibilities offered by optofluidics. Tunability is also used for other optofluidic elements, in particular for tunable laser sources [78-87].

Furthermore, the basic elements of optical platforms have been developed with optofluidic principles, namely waveguides [88-94] and optical switches [95-99]. The optofluidic switch has even reached industrial level (Agilent Photonic Switching Platform).
For implementation of an operational optofluidic analysis systems further optimization is required. In the beginning of the optofluidic era Psaltis et al. introduced a general platform concept, built of three functional layers, (1) an optical layer, (2) a microfluidic channel layer, and (3) a fluid control layer. In order to realize such a design and thus to pave the way for optofluidic products on the market, the individual components are to be combined to an entire system.

\section{Conclusion}

In this manuscript, we discussed modular platforms for the interconnection of optofluidic devices to a total analysis system. We believe that modular concepts and welldefined coupling interfaces would promote industrial implementation of optofluidic systems. In particular, the development process of product families would benefit from this approach.

\section{Acknowledgement}

The authors thank C. Megnin from the Institute of $\mathrm{Mi}-$ crostructure Technology (IMT) of the Karlsruhe Institute of Technology (KIT) for the development and supply of microvalves, D. G. Rabus from Festo AG \& Co. KG, for the inspiring discussions, as well as A. Voigt, S. Sobich, and C. Essig from the IMT of the KIT, C. Schmuck from Bürkert Fluid Control Systems, H. Besser from the Institute of Applied Material Research of the KIT, and A. Hofmann from the Institute for Applied Computer Science of the KIT for technical support. This work has been supported by the Bürkert Technology Center, Karlsruhe.

\section{References}

[1] D. Psaltis, S. R. Quake, and C. Yang, "Developing optofluidic technology through the fusion of microfluidics and optics," Nature, vol. 442, pp. 381-386, 2006.

[2] C. Monat, R. Comachuk, and B. J. Eggleton, "Integrated optofluidics: A new river of light," Nature Photon., vol. 1, pp. 106-114, 2007.

[3] Y. Fainman, L. P. Lee, D. Psaltis, C. Yang, Optofluidics: Fundamentals, Devices, and Applications, McGraw Hill, New York, US, 2009.

[4] A. R. Hawkins, H. Schmidt, Handbook of Optofluidics, CRC Press, Boca Raton, FL, US, 2010.

[5] X. Fan and I. M. White, "Optofluidic microsystems for chemical and biological analysis," Nature Photon., vol. 5, pp. 591-597, 2011.

[6] L. Pang, Y. Fainman, H. M. Chen and L. M. Freeman, "Optofluidic devices and applications in pho- 
tonics, sensing and imaging," Lab Chip, vol. 12, pp. 3543-3551, 2012.

[7] A. Manz, N. Graber and H. Widmer, "Miniaturized total chemical analysis systems: a novel concept for chemical sensing," Sens. Acuators B, vol. 1, pp. 244248, 1990.

[8] P. Gravesen, J. Branebjerg, and O. S. Jensen, "Microfluidics-a review," J. Micromech. Microeng., vol. 3, pp. 143-164, 1993.

[9] G. M. Whitesides, "The origins and the future of microfluidics," Nature, vol. 442, pp368-373, 2006.

[10] S. C. Terry, J. H. Hermann and J. B. Angel "A gas chromatographic air analyzer fabricated on a silicon wafer," IEEE Trans. Electron Devices, vol. 26, pp. 1880-1886, 1979.

[11] E. Verpoorte, A. Manz, H. Lüdi, A. E. Bruno, F. Maystre, B. Krattiger, H. M. Widmer, "A silicon flow cell for optical detection in miniaturized total chemical analysis systems," Sens. Actuators B, vol. 50, pp. 66-70, 1992

[12] R. Zengerle, S. Kluge, M. Richter, and A. Richter, "A bidirectional silicon micropump," Sens. Actuators A, vol. 50, pp. 81-86, 1995.

[13] J. C. McDonald, D. C. Duffy, J. R. Anderson, D. T. Chiu, H. Wu, O. J. A. Schueller, G. M. Whitesides, "Fabrication of microfluidic systems in poly(dimethylsiloxane)," Electrophoresis, vol. 21, pp. 27-40 2000.

[14] J. C. McDonald and G. M. Whitesides, "PDMS as a material for fabricating microfluidic devices," Acc. Chem. Res., vol. 35, pp. 491-499, 2002.

[15] H. Becker, C. Gärtner, "Polymer microfabrication technologies for microfluidic systems," Anal. Bioanal. Chem., vol. 390, pp. 89-111, 2008.

[16] E. Berthier, E. W. K. Young, and D. Beebe, "Engineers are from PDMS-land, Biologists are from Polystyrenia," Lab Chip, vol. 12, pp. 1224-1237, 2012.

[17] T. D. Boone, Z. H. Fan, H. H. Hooper, A. J. Ricco, H. Tan, and S. J. Williams, "Plastic Advances Microfluidic Devices," Anal. Chem., vol. 1, pp. 78A-86A, 2002.

[18] G. S. Fiorini and D. T. Chiu, "Disposable microfluidic devices: fabrication, function, and application," Bio Techniques, vol. 38, pp. 429-446, 2005.

[19] Y.-C. Su, J. Shah, and L. Lin, "Implementation and Analysis of Polymeric Microstructures Replication by Micro Injection Molding," J. Micromech. Microeng., vol. 14, pp. 415-422, 2004.

[20] M. Worgull, A. Kolew, M. Heilig, M. Schneider, H. Dinglreiter, B. Rapp, "Hot embossing of high performance polymers," Microsyst. Technol., vol. 17, pp. 585-592, 2011.

[21] C. D. Chin, V. Linder and S. K. Sia, "Commercialization of microfluidic point-of-care diagnostic devices," Lab Chip, vol. 12, pp. 2118-2134, 2012.

[22] A. W. Martinez, S. T. Phillips, and G. M. Whitesides, "Three-dimensional microfluidic devices fabricated in layered paper and tape," Proc. Natl Acad. Sci. USA, vol. 105, 19606-19611, 2008

[23] D. R. Ballerini, X. Li, and W. Shen, "Patterned paper and alternative materials as substrates for lowcost microfluidic diagnostics," Microfluid. Nanofluid., 2012, DOI: 10.1007/s10404-012-0999-2.

[24] C. W. Tsao and D. L. DeVoe, "Bonding of thermoplastic polymer microfluidics," Microfluid. Nanofluid., vol. 6, pp. 1-16, 2009.

[25] W. Pfleging and O. Baldus, "Laser patterning and welding of transparent polymers for microfluidic device fabrication," Proc. SPIE, vol. 6107, pp. 61070512, 2006.

[26] F. J. Kamphoefner, "Ink jet printing," IEEE Transact. Electron Devices, vol 19., pp. 584-593, 1998.

[27] W. E. Haas, "Liquid-crystal display research - the first 15 years," Mol. Cryst. Liq. Cryst., vol. 94, pp. 1-31, 1983.

[28] P. S. Drzaic, "Polymer dispersed nematic liquid crystal for large area displays and light valves," J. Appl. Phys., vol. 60, pp. 2142-2148, 1986.

[29] Frost and Sullivan, "Lab on a Chip (LOC) - Advances in Microfluidics," Market Analysis, www.frost.com, 2004.

[30] Yole Développement, "Emerging Markets For Microfluidic Applications", Market Analysis, www . i-micronews. com, 2011.

[31] H. van Heeren, "Standards for connecting microfluidic devices?," Lab Chip, vol. 12, pp. 1022-1025, 2012.

[32] H. Becker, "Hype, hope and hubris: the quest for the killer application in microfluidics," Lab Chip, vol. 9, pp. 2119-2122, 2009.

[33] R. Marie, A. Kristensen, "Nanofluidic devices towards single DNA molecule sequence mapping," J. Biophoton., vol. 5, pp. 673-686, 2012.

[34] J. Wu, G. Zheng and L. M. Lee, "Optical imaging techniques in microfluidics and their applications," Lab Chip, vol. 12, pp. 3566-3575, 2012.

[35] M. Brammer, C. Megnin, A. Voigt, M. Kohl, and T. Mappes, "Modular Optoelectronic Microfluidic Backplane for Total Analysis Systems," J. Microelectromech. S., accepted for publication.

[36] N. Z. Danckwardt, M. Franzreb, A. E. Guber, V. Saile, "Pump-free transport of magnetic particles in microfluidic channels," J. Magn. Magn. Mater., vol. 323, pp. 2776-2781, 2011.

[37] J. Ducrée, S. Haeberle, S. Lutz, S. Pausch, F. Von Stetten and R. Zengerle, "The centrifugal microfluidic 
Bio-Disk platform," J. Micromech. Microeng., vol. 17, pp. S103-115, 2007.

[38] R. B. Fair, "Digital microfluidics: Is a true lab-ona-chip possible?," Microfluid. Nanofluid., vol. 3, pp. 245-281, 2007.

[39] K. Chakrabarty, R. B. Fair, and J. Zeng, "Design Tools for Digital Microfluidic Biochips: Toward Functional Diversification and More than Moore," IEEE Trans. Comput. Aided Des. Integrated Circ. Syst., vol. 29, pp. 1001-1016, 2010.

[40] K. W. Oh and C. H. Ahn, "A review of microvalves," J. Micromech. Microeng., vol. 16, pp. R13-R39, 2006.

[41] T. Grund, C. Megnin, J. Barth, and M. Kohl, "Batch fabrication of shape memory actuated polymer microvalves by transfer bonding techniques," J. Microelectron. Electron. Packaging, vol. 6, pp. 219-227, 2009.

[42] C. Megnin, M. Brammer, H. Luckert, and M. Kohl, "SMA Microvalves with Plug in Interface for a Modular Fluidic Backplane," Actuator, 18.-20.6.2012, Bremen, 2012.

[43] M. A. Unger, H. P. Chou, T. Thorsen, A. Scherer, S. R. Quake, "Monolithic microfabricated valves and pumps by multilayer soft lithography," Science, vol. 288, pp. 113-116, 2000.

[44] B. E. Rapp, L. Carneiro, K. Länge, and M. Rapp, "An indirect microfluidic flow injection analysis (FIA) system allowing diffusion free pumping of liquids by using tetradecane as intermediary liquid," Lab Chip, vol. 9, pp. 354-356, 2009.

[45] B. Mosadegh, T. Bersano-Begey, J. Y. Park, M. A. Burns, and S. Takayama, "Next-generation integrated microfluidic circuits," Lab Chip, vol. 11, pp. 28132818, 2011.

[46] P. K. Yuen, J. T. Bliss, C. C. Thompson, and R. C. Peterson, "Multidimensional modular microfluidic system," Lab Chip, vol. 9, pp. 3303-3305, 2009.

[47] G. Perozziello, G. Simone, P. Candeloro, F. Gentile, N. Malara, R. Larocca, M. Coluccio, S. A. Pullano, L. Tirinato, O. Geschke, and E. Di Fabrizio, "A Fluidic Motherboard for Multiplexed Simultaneous and Modular Detection in Microfluidic Systems for Biological Application," Micro Nanosys., vol. 2, pp. 227238, 2010.

[48] S. M. Langelier, E. Livak-Dahl, A. J. Manzo, B. N. Johnson, N. G. Walter, and M. A. Burns, "Flexible casting of modular self-aligning microfluidic assembly blocks," Lab Chip, vol. 11, pp. 1679-1688, 2011.

[49] M. Brammer, C. Megnin, T. Parvanta, M. Siegfarth, T. Mappes, and D. G. Rabus, "A modular microfluidic backplane for control and interconnection of optofluidic devices," IEEE Winter Topicals, pp. 101-102,
2011.

[50] A. M. Christensen, D. A. Chang-Yen, and B. K. Gale, "Characterization of interconnects used in PDMS microfluidic systems," J. Micromech. Microeng., vol. 15, pp. 928-934, 2005.

[51] R. Lo and E. Meng, "Reusable, adhesiveless and arrayed in-plane microfluidic interconnects," J. Micromech. Microeng., vol. 21, pp. 025021-025035, 2011.

[52] C. González, S. D. Collins, and R. L. Smith, "Fluidic Inter-connects for Modular Assembly of Chemical Microsystems," Sens. Actuators B, vol. 49, pp. 40-45, 1998.

[53] G. Perozziello, F. Bundgaard, O. Geschke, "Fluidic inter-connections for microfluidic systems: A new integrated fluidic interconnection allowing plug 'n' play functionality," Sens. Actuators B, vol. 130, pp. 947953, 2008

[54] V. Nittis, R. Fortt,C. H. Legge and A. J. De Mello, "A high-pressure interconnect for chemical microsystem applications," Lab Chip, vol. 1, pp. 148-152, 2001.

[55] L. Eldada and L. W. Shacklette, "Advances in polymer integrated optics," IEEE J. Sel. Topics Quantum Electron., vol. 6, no. 1, pp. 54-68, 2000.

[56] H. Ma, A. K.-Y. Jen, and L. R. Dalton, "Polymer-Based Optical Waveguides: Materials, Processing, and Devices," Adv. Mater., vol. 14, pp. 1339-1365, 2002.

[57] D. G. Rabus, P. Henzi, and J. Mohr, "Photonic Integrated Circuits by DUV-Induced Modification of Polymers," IEEE Photon. Tech. Lett., vol. 17, pp. 591593, 2005.

[58] D. G. Rabus, M. Bruendel, Y. Ichihashi, A. Welle, R. A. Seger, and M. Isaacson, "A Bio-Fluidic-Photonic Platform Based on Deep UV Modification of Polymers," IEEE J. Sel. Topics Quantum Electron., vol. 13, pp. 214-222, 2007.

[59] J. Halldorsson, N. B. Arnfinnsdottir, A. B. Jonsdottir, B. Agnarsson, and K. Leosson, "High index contrast polymer waveguide platform for integrated biophotonics," Opt. Express, vol. 18, pp. 16217-16226, 2010.

[60] T. Mappes, C. Vannahme, M. Schelb, U. Lemmer, J. Mohr, "Design for optimized coupling of organic semiconductor laser light into polymer waveguides for highly integrated biophotonic sensors," Microelectron. Eng., vol 86, pp. 1449-1501, 2009.

[61] L. Y. Lin, E. L. Goldstein, and R. W. Tkach, "Freespace micromachined optical switches with submillisecond switching time for large-scale optical crossconnects," IEEE Photon. Technol. Lett., vol. 10, pp. 525-527, 1998

[62] T. Yamamoto, J. Yamaguchi, N. Takeuchi, A. Shimizu, E. Higurashi, R. Sawada, Y. Uenishi, "A three- 
dimensional MEMS optical switching module having 100 input and 100 output ports," IEEE Photon. Technol. Lett., vol. 10, pp. 1360-1362, 2003.

[63] M. Brammer, C. Megnin, M. Siegfarth, S. Sobich, A. Hofmann, D. G. Rabus, and T. Mappes, "Optofluidic backplane as a platform for modular system design," Proc. SPIE, vol. 8251, pp. 825100-8, 2012.

[64] U. Wallrabe, H. Dittrich, G. Friedsam, T. Hanemann, J. Mohr, K. Müller, V. Piotter, P. Ruther, T. Schaller, W. Zissler, "Micro-molded easy-assembly multi fiber connector: RibCon§," Microsyst. Technol., vol. 8, pp. 83-87, 2002.

[65] M. Brammer, M. Siegfarth, T. Mappes, "Optical coupling," Patent application DE102012004656, US13421485 (15.3.2011).

[66] R. Turton, Analysis, synthesis, and design of chemical processes, Prentice Hall, Upper Saddle River, NJ, US, 3. Edt., 2009.

[67] K. A. Bakeev, Process analytical technology, Blackwell, Oxford, UK, 2. Edt., 2010.

[68] U. Levy, R. Shamai, "Tubable optofluidic devices," Microfluid. Nanofluid., vol. 4, pp. 97-105, 2008.

[69] N.-T. Nguyen, "Micro-optofluidic Lenses: A review," Biomicrofluid., vol. 4, pp. 031501-15, 2010.

[70] B. Berge, J. Peseux, "Variable focal lens controlled by an external voltage: an application of electrowetting," Eur. Phys. J. E, vol. 3, pp. 159-163, 2000.

[71] T. Krupenkin, S. Yang, and P. Mach, "Tubable liquid microlens," Appl. Phys. Lett., vol. 82, pp. 316-318, 2003.

[72] S. Kuiper and B. H. W. Hendriks, "Variable-focus liquid lens for miniature cameras," Appl. Phys. Lett., vol. 85, pp. 1128-1130, 2004.

[73] L. Dong and H. R. Jiang, "Variable-focus liquid lens for miniature cameras," J. Microelectromech. S., vol. 17, pp. 381-392, 2008.

[74] J. J. Shi, Z. Stratton, S. C. S. Lin, H. Huang, and T. J. Huang, "Tunable optofluidic microlens through active pressure control of an air-liquid interface," Microfluid. Nanofluid., vol. 9, pp. 313-318, 2010.

[75] D. Y. Zhang, V. Lien, Y. Berdichevsky, J. H. Choi, and Y. H. Lo, "Fluidic adaptive lens with high focal length tenability," Appl. Phys. Lett., vol. 82, pp. 3171-3173, 2003.

[76] A. Werber and H. Zappe, "Tubable microfluidic microlenses," Appl. Opt., vol. 44, pp. 3238-3245, 2005.

[77] H. Ren and S.-T. Wu, "Variable-focus liquid lens," Opt. Express, vol. 15, pp. 5931-5936, 2007.

[78] D. V. Vezenov, B. T. Mayers, R. S. Conroy, G. M. Whitesides, P. T. Snee, Y. Chan, D. G. Nocera, and M. G. Bawendi, "A Low-Threshold, High-Efficiency Microfluidic Waveguide Laser," J. Am. Chem. Soc., vol.
127, pp. 8952-8953, 2005.

[79] J. C. Gala, J. Torres, M. Belotti, Q. Kou, and Y. Chen, "Microfluidic tunable dye laser with integrated mixer and ring resonator," Appl. Phys. Lett., vol. 86, pp. 264101-3, 2005.

[80] Z. Y. Li, Z. Y. Zhang, T. Emery, A. Scherer, and D. Psaltis, "Single mode optofluidic distributed feedback dye laser," Opt. Express, vol. 14, pp. 696-701, 2006

[81] M. Gersborg-Hansen and A. Kristensen, "Tunability of optofluidic distributed feedback dye lasers," Opt. Express, vol. 15, pp. 137-142, 2007.

[82] S. Lacey, I. M. White, Y. Sun, S. I. Shopova, J. M. Cupps, P. Zhang, and X. Fan, "Versatile opto-fluidic ring resonator lasers with ultra-low threshold," Opt. Express, vol. 15, pp. 15523-15530, 2007.

[83] J. D. Suter, Y. Sun, D. J. Howard, J. A. Viator, and $X$. Fan, "PDMS embedded opto-fluidic microring resonator lasers," Opt. Express, vol. 16, pp. 1024810253, 2008.

[84] C. Vannahme, M. B. Christiansen, T. Mappes, and A. Kristensen, "Optofluidic dye laser in a foil," Opt. Express, vol. 18, pp. 9280-9285, 2010.

[85] W. Song and D. Psaltis, "Pneumatically tunable optofluidic dye laser," Appl. Phys. Lett., vol. 96, pp. 081101-3, 2010.

[86] Y. Yang , A. Q. Liu , L. Lei , L. K. Chin , C. D. Ohl , Q. J. Wang and H. S. Yoon, "A tunable 3D optofluidic waveguide dye laser via two centrifugal Dean flow streams," Lab Chip, vol. 11, pp. 3182-3187, 2011.

[87] T. Wienhold, F. Breithaupt, C. Vannahme, M. B. Christiansen, W. Dörfler, A. Kristensen and T. Mappes "Diffusion driven optofluidic dye lasers encapsulated into polymer chips," Lab Chip, DOI: 10.1039/C2LC40494J.

[88] D. B. Wolfe, R. S. Conroy, P. Garstecki, B. T. Mayers, M. A. Fischbach, K. E. Paul, M. Prentiss, and G. M. Whitesides, "Dynamic control of liquid-core/liquidcladding optical waveguides," Proc. Ntl Acad. Sci. USA, vol. 101, pp. 12434-12438, 2004.

[89] P. Measor, S. Kühn, E. J. Lunt, B. S. Phillips, A. R. Hawkins, and H. Schmidt, "Hollow-core waveguide characterization by optically induced particle transport," Opt. Lett., vol. 33, pp. 672-674, 2008.

[90] H. Schmidt and A. R. Hawkins, "Optofluidic waveguides: I. Concepts and implementations," Microfluid. Nanofluid., vol. 4 pp. 3-16, 2008.

[91] A. R. Hawkins and H. Schmidt, "Optofluidic waveguides: II. Fabrication and structtures," Microfluid. Nanofluid., vol. 4 pp. 17-32, 2008.

[92] S. H. Cho, "Optofluidic Waveguides in Teflon AFCoated PDMS Microfluidic Channels," IEEE Phton. Technol. Lett., vol. 21, pp. 1057-1059, 2009.

[93] J. D. Suter, W. Lee, D. J. Howard, E. Hoppmann, I. M. 
White, and X. Fan, "Demonstration of the coupling of optofluidic ring resonator lasers with liquid waveguides," Opt. Lett., vol. 35, pp. 2997-2999, 2010.

[94] A. J. Chung and D. Erickson, "Optofluidic waveguides for reconfigurable photonic systems," Opt. Express, vol. 19, pp. 8602-8609, 2011.

[95] N.-T. Nguyen, T.-F. Kong, J.-H. Goh, and C. L.-N. Low, "A micro optofluidic splitter and switch based on hydrodynamic spreading," J. Micromech. Microeng., vol. 17, pp. 2169-2174, 2007.

[96] A. Groisman, S. Zamek, K. Campbell, L. Pang, U. Levy, and Y. Fainman, "Optofluidc 1x4 Switch," Opt. Ex- press, vol. 16, pp. 13499-13508, 2008.

[97] B.-T. Liao, H.-H. Shen, H.-H. Liao, and Y.-J. Yang, "A bi-stable 2x2 optical switch monolithically integrated with variable optical attenuators," Opt. Express, vol. 17, pp. 19919-19925, 2009.

[98] A. H. J. Yang and D. Erickson, "Optofluidic ring resonator switch for optical particle transport," Lab Chip, vol. 10, pp. 769-774, 2010.

[99] W. Song and D. Psaltis, "Pneumatically tunable optofluidic 2x2 switch for reconfigurable optical ciruit," Lab Chip, vol. 11, pp. 2397-2402, 2011. 